\title{
Sublingual immunotherapy for Japanese cedar pollinosis: potential biomarkers predicting therapeutic responses
}

\author{
Satoru Masuno and Kimihiro Okubo* \\ Department of Otolaryngology, Nippon Medical School, Tokyo, Japan
}

\begin{abstract}
Objective: Sublingual immunotherapy (SLIT) for allergic rhinitis due to Japanese cedar (JC) pollisosis was investigated to examine its clinical efficacy and adverse effects, as well as the changes of serum antibodies.

Research design and methods: A placebo-controlled, double-blind study was performed with 12 patients in the SLIT group and 16 patients in the placebo group. Treatment was started before the JC pollen season and was continued until after the pollen season. The SLIT group received daily up-titration of a JC pollen allergen extract over 2 weeks, followed by maintenance therapy twice weekly. The placebo group received the vehicle of the allergen extract at the same dosage. Subjects kept symptom diaries during the JC pollen season.
\end{abstract}

Main outcome measures: Scores were assigned for nasal symptoms (sneezing, itching, rhinorrhea, and obstruction) and the total nasal symptom score (TNSS) was calculated during the pollen season. Immunoglobulin levels were measured before and after treatment.

Results: During the 90-day period from February 1 to April 30, the sneezing score, nasal itching score, rhinorrhea score, nasal congestion score, and TNSS were significantly lower in the SLIT group than the placebo group for 15, 15, 33, 22, and 37 days, respectively.

Serum levels of JC allergen-specific IgG and IgE showed no significant difference between the 2 groups before treatment, but were significantly higher in the SLIT group than the placebo group after the pollen season. Although JC allergen-specific IgG4 was also higher in the SLIT group after the pollen season, there was no significant difference. The IgG4/IgG ratio showed no significant difference between the two groups either before treatment or after the pollen season. Adverse events were only Grade 1 in both groups and resolved spontaneously.

Conclusions: SLIT suppressed symptoms of JC pollinosis and increased the JC-specific IgG4/IgG ratio in the first season of treatment.

\section{Introduction}

Allergic rhinitis/pollinosis (hay fever) was first reported by Blackley [1] in 1873. In Japan, Saito [2] first reported Japanese cedar (JC) pollinosis in 1964, and it subsequently increased owing to large-scale planting of cedar trees during the 1970s. A survey performed in 2008 showed that the prevalence of JC pollinosis was $26.5 \%$ in Japan [3], making it the major allergic disease affecting the Japanese population.

Noon initially reported the effectiveness of immunotherapy for allergy in 1911[4]. Immunotherapy for allergic diseases has subsequently attracted considerable attention in many countries including Japan. In the 1960s, a pollen-specific antigen extract became available for immunotherapy in Japan and a standardized cedar antigen-specific extract was released in 2000 .

Because of various problems associated with subcutaneous immunotherapy (SCIT), including pain during injection, frequent visits for treatment, and side effects such as anaphylaxis, other methods of delivering immunotherapy have been examined. In Western countries, sublingual immunotherapy (SLIT) has achieved good results for ragweed allergy and gained popularity, while SLIT using standardized specific cedar antigen extract has also been initiated in Japan. The response rate to SCIT is believed to be better than $70 \%$, and the response rate is even higher in persons with sensitization to cedar pollen alone. However, approximately two years of treatment is required. Also, although investigations have been performed to identify biomarkers that can predict the response before or at an early stage of treatment, no markers have been identified so far.

Immunotherapy induces both cellular and humoral regulatory mechanisms. Interleukin(IL)-10, a cytokine expressed after immunotherapy by nasal mucosal and peripheral blood regulatory $\mathrm{T}$ cells, play an important role on cellular mechanism in inhibition of T-cell cytokine production and activation of mast cells and eosinophils [5]. On the other hand, allergen specific antibodies, especially of the IgG4 isotype, play an important role on humoral mechanism as an blocking antibodies in inhibition of IgE-mediated histamine release and antigen presentation through inhibitory FcrR II b IgG receptors [6].

Serum cedar allergen-specific IgG4 is very small amount and how the value changes in SLIT treatment period is still unknown. We tried the measurement of IgG4 and the comparison of the IgG4/IgG ratio to confirm that IgG4, included in very small amount in total IgG, increased

Correspondence to: Dr. Kimihiro Okubo, Department of Otolaryngology, Nippon Medical School, 1-1-5 Sendagi Bunkyo-ku, Tokyo 113-8603, Japan; Tel: +81 035814 6213; Fax: +81 035814 6207; E-mail: ent-kimi@nms.ac.jp

Key words: sublingual immunotherapy, specific serum antibody, Japanese cedar pollinosis, placebo-controlled, double-blind study, total nasal symptom score

Received: July 14, 2016; Accepted: August 08, 2016; Published: August 12, 2016 
by SLIT and examined the correlation with clinical manifestations.

Against this background, we conducted a placebo-controlled, double-blind study of SLIT for JC pollinosis to examine its clinical efficacy and adverse effects, as well as to determine the changes in the serum levels of specific antibodies for JC allergen.

\section{Patients and methods}

\section{Subjects}

We performed a placebo-controlled, double-blind study of SLIT for JC pollinosis. Observation was initiated in November 2011 (before the 2012 cedar pollen season) and was continued until May 2012 (after the pollen season). The subjects were patients with cedar pollen allergy aged 15 years or older who lived in Tokyo and the surrounding area. JC pollinosis was diagnosed if JC-specific IgE was class 2 or higher by the CAP-RAST method (Phadia, Tokyo, Japan) and clinical manifestations coincided with the cedar pollen season [7].

Based on the standard exclusion criteria for SCIT, patients were excluded from this study for the following reasons: serious progressive disease, use of systemic steroids, anticancer drugs, or beta-stimulants, severe asthma, pregnancy, a history of immunotherapy or sinus surgery, and other nasal complications.

The enrolled subjects were randomly divided into 2 groups (true medicine group and placebo group) with a ratio of 1:1 by the number that a patient was given first, and after completion of treatment the number key was opened by a controller who was not directly involved in this study.

\section{Ethical considerations}

This study was conducted in accordance with the Declaration of Helsinki. All of the subjects gave written informed consent to participation.

\section{Study design}

The study design is shown in Figure 1 and 2. After the pollen season finished in 2011, the study was explained to candidate patients and those who gave consent were registered. Blood samples were collected in early November 2011, after which the subjects were assigned to the

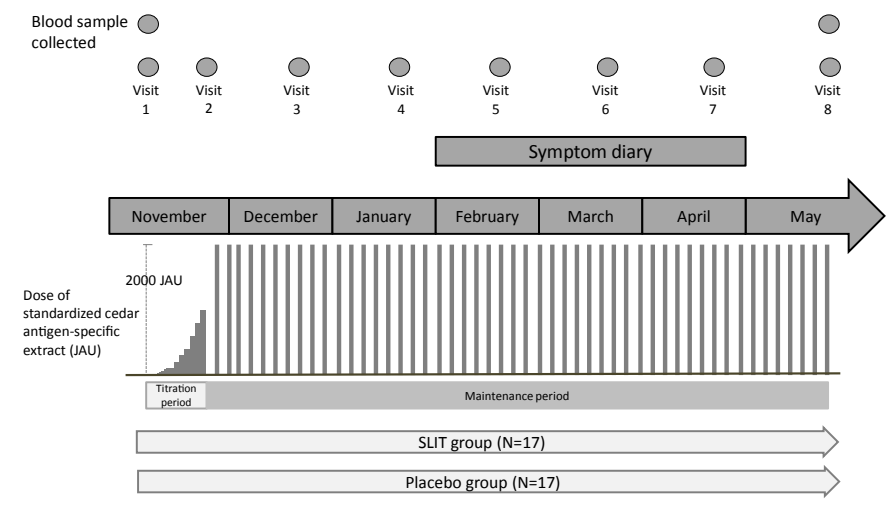

Figure 1. Outline of the study

After a blood sample was collected in early November, the subjects were assigned to the SLIT group or the placebo group and treatment was started (Visit 1). The dose of antigen extract was titrated during daily administration for 2 weeks. Then adverse events were checked, and twice weekly administration of cedar pollen allergen extract $(2,000 \mathrm{JAU} / \mathrm{mL})$ was started (Visit 2). Thereafter, patients were reviewed every month (Visits 3-7), and were asked to record symptoms in a diary from February to April (pollen season). A second blood sample was collected in May and administration was finished (Visit 8).

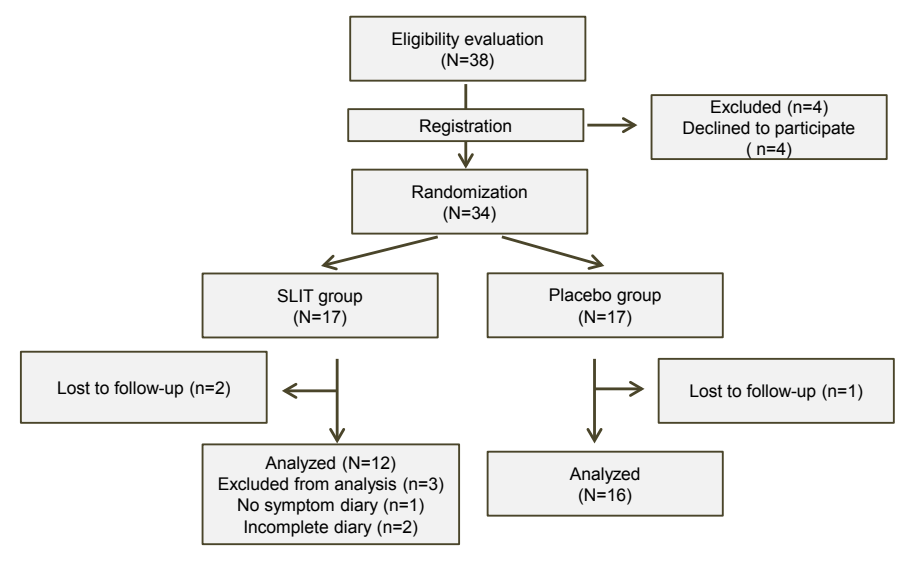

Figure 2. CONSORT flow diagram

Out of 38 candidate subjects, 4 refused to participate and 34 patients were registered, after which 17 patients were randomized to each group. Two subjects in the SLIT group and 1 subject in the placebo group were lost to follow-up. Three subjects were excluded from analysis in the SLIT group (1refused to record symptoms in the symptom diary and 2 had incomplete diaries).

Table 1. Dosing schedule of the SLIT group.

\begin{tabular}{|c|c|c|c|}
\hline & $\begin{array}{c}\text { Week 1 } \\
\text { (200 JAU/mL) }\end{array}$ & $\begin{array}{c}\text { Week 2 } \\
\text { (2000 JAU/mL) }\end{array}$ & $\begin{array}{c}\text { Week 3 onward } \\
\text { (2000 JAU/mL) }\end{array}$ \\
\hline Day 1 & 1 drop & 1 drop & 20 drops \\
\hline Day 2 & 2 drops & 2 drops & \\
\hline Day 3 & 3 drops & 3 drops & \\
\hline Day 4 & 4 drops & 4 drops & \\
\hline Day 5 & 6 drops & 6 drops & 20 drops \\
\hline Day 6 & 8 drops & 8 drops & \\
\hline Day 7 & 10 drops & 10 drops & \\
\hline
\end{tabular}

20 drops $=1 \mathrm{~mL}$

A standardized allergen extract (200 JAU/mL and 2,000 JAU/mL) of JC was administered to the SLIT group. Treatment was delivered by using a special dispenser that delivered drops with a volume of $50 \mu \mathrm{g}$. Dose titration was performed in Weeks 1 and 2, while a stable dose of 20 drops $(1 \mathrm{~mL})$ was administered twice a week from Week 3 onward. The same volume of $50 \%$ glycerin was administered to the placebo group.

SLIT group or the placebo group and treatment was started (Visit 1). JC allergen extract (Therapeutic Standardized Allergen Extract for Subcutaneous Injection Torii Cedar Pollen, Torii Pharmaceutical, Co. Ltd., Tokyo, Japan) was administered to the SLIT group and 50\% glycerin (the vehicle of this allergen extract) was given to the placebo group. The dose of allergen extract was up-titrated during daily administration for the initial 2 weeks of the study according to the schedule in Table 1 by using a special dispenser that delivered drops with a volume of $50 \mu \mathrm{g}$. The method of administration was sublingual swallow, in which drops of the drug were placed under the tongue and retained for 2 minutes before being swallowed. Subjects were observed while taking the first dose of the extract, which had strength of 200 Japanese Allergy Units (JAU)/mL, and they subsequently took the drug at home, switching to a stronger extract $(2,000 \mathrm{JAU} / \mathrm{mL})$ in Week 2. Adverse effects were checked after 2 weeks (Visit 2). If it was determined that the study could be continued, administration of the allergen extract $(2,000 \mathrm{JAU} / \mathrm{mL})$ was continued twice weekly. Thereafter, examination was conducted every month (Visits 3-7), and the subjects were asked to record their symptoms in a diary from February to April (cedar pollen season). Blood samples were collected in May (Visit 8), and administration was finished. The total dose of allergen extract administered during the study was approximately 106,400 JAU. The placebo group received the same volume of $50 \%$ glycerin for each dose, which was indistinguishable from the allergen extract. 
If symptoms occurred during the cedar pollen season, secondgeneration oral antihistamines were permitted for nasal symptoms and second-generation antihistamine eye drops could be used for ocular symptoms that severely interfered with daily activities.

\section{Assessment of symptoms}

Symptoms were recorded in a symptom diary for 90 days from February 1 to April 30, 2012 in the JC pollen season. Scores were assigned for sneezing, nasal itching, rhinorrhea, and nasal obstruction, and then were summed to calculate the total nasal symptom score (TNSS). The number of sneezing episodes was recorded 3 times a day (morning, afternoon, and evening). Then a score was assigned as follows according to the daily number of sneezing attacks: no attacks = $0 ; 1-5$ attacks $=1 ; 6-10$ attacks $=2 ; 11-20$ attacks $=3$; and 21 or more attacks $=4$. Subjects were instructed to record continuous sneezing as a single attack, while sneezing that occurred after an interval was regarded as a separate attack. To assess nasal itching, the number of times the nose was scratched because of an itchy sensation was recorded 3 times a day (morning, afternoon, and evening). Then a score was assigned by the same method as that used for sneezing episodes. Subjects were instructed to record continuous scratching of the nose as a single episode, while scratching that occurred after an interval was regarded as a separate episode. To assess rhinorrhea, the number of times of blowing the nose was also recorded 3 times a day (morning, afternoon, and evening), and a score was assigned as follows according to the daily number of times the nose was blown: no times $=0 ; 1-5$ times $=1 ; 6-10$ times $=2 ; 11-20$ times $=3$; and 21 or more times $=4$. Nasal obstruction was also assessed 3 times a day (morning, afternoon, and evening) and a score was assigned as follows according to the severity of obstruction: none $=0$; obstruction not requiring mouth breathing $=1$; severe obstruction $=2$; very severe obstruction $=3$; and constant complete obstruction $=4$. Then the mean daily score for nasal obstruction was calculated from the morning, afternoon, and evening scores and it was assigned a score as follows: $0=0$ points; $>0$ and $\leq 1$ $=1$ point $>1$ and $\leq 2=2$ points, $>2$ and $\leq 3=3$ points, and $>3$ and $\leq$ $4=4$ points.

\section{Measurement of serum cedar allergen-specific antibodies}

Peripheral blood samples were collected from all subjects in November 2011 before treatment was started and in May 2011 when treatment finished after the end of the pollen season. Serum was separated and stored at $-80^{\circ} \mathrm{C}$ until measurement of the serum levels of cedar allergen-specific IgG, IgG4, and IgE using an allergy specific IgE assay kit (AlaSTAT $3 \mathrm{~g}$ ), which were conducted at Siemens Healthcare Diagnostics K.K.( Tokyo, Japan). In addition, the cedar allergen-specific IgG4/IgG ratio was calculated to examine changes in the production of IgG4, which is considered to be a blocking antibody, relative to IgG.

\section{Statistical analysis}

The $t$-test was used to investigate the significance of differences in patient characteristics, except that Fisher's exact test was used to compare gender. The $t$-test was also used to test the significance of difference in symptoms (sneezing, nasal itch, rhinorrhea, nasal obstruction, and TNSS). Fisher's exact test was also used to determine whether there was a significant difference in the number of days when symptoms showed significant improvement during the pollen season. Finally, the $t$-test was used to identify significant differences in the serum levels of cedar allergen-specific antibody and the IgG4/IgG ratio between before treatment and after the pollen season as well as to test changes of antibody levels.

\section{Results}

\section{Characteristics of the patients}

Thirty-eight patients were recruited as candidate subjects and considered participating in the study. Four patients refused to participate after receiving an explanation of the study and the remaining 34 patients were randomized (17 to the SLIT group and 17 to the placebo group). Two subjects in the SLIT group and 1 subject in the placebo group did not complete all the study visits; hence, 15 subjects in the SLIT group and 16 subjects in the placebo group completed the study. One subject in the SLIT group refused to use the symptom diary and 2 subjects in this group had incomplete diaries, and analysis was conducted in 12 subjects from the SLIT group and 16 subjects from the placebo group (Figure 2). Table 2 shows the characteristics of each group. There were no significant differences between the two groups with respect to the age, sex, eosinophil count at diagnosis, total IgE, and the RAST class and actual RAST values for cedar pollen, cypress pollen, house dust, mites, and ragweed.

\section{Airborne pollen count}

In Tokyo (Chiyoda Ward), the pollen count during the 2012 cedar pollen season was measured as $1252.2 / \mathrm{cm}^{2}$ by a Durham standard pollen sampler. The season started on March 3 and the maximum pollen count was detected on March 7 (159.3/cm).

\section{Symptoms}

Figure 3 displays data on the scores for sneezing, nasal itching, rhinorrhea, and nasal congestion, as well as the TNSS, in both groups. During the 90-day period from February 1 to April 20, 2012, the sneezing score, nasal itching score, rhinorrhea score, nasal congestion score, and TNSS were significantly lower in the SLIT group than the placebo group for 15 days ( $16.7 \%$ of the observation period), 15 days (16.7\%), 33 days (36.7\%), 22 days (24.4\%), and 37 days (41.1\%), respectively. During the actual pollen season (42 days from March 3 to April 13), these scores were significantly lower in the SLIT group than the placebo group for 14 days (33.3\% of the pollen season), 9 days (21.4\%), 25 days (59.5\%), 19 days (45.2\%), and 28 days (66.7\%), respectively. Except for nasal itching, the symptom scores were lower in the SLIT group than

Table 2. Characteristics of the patients.

\begin{tabular}{|l|c|c|c|}
\hline & Placebo group & SLIT group & P value \\
\hline $\mathrm{N}$ & 16 & 12 & \\
\hline Age & $53.9(25-74)$ & $47.3(22-63)$ & 0.0755 \\
\hline Sex (male/female) & $(9 / 7)$ & $(5 / 7)$ & $0.704^{*}$ \\
\hline Eosinophils (\%) & $2.86(0.4-6.6)$ & $2.99(1.1-4.7)$ & 0.404 \\
\hline Total IgE & $107(34-328)$ & $74.7(5-287)$ & 0.160 \\
\hline Cedar pollen (class) & $3.31(3-4)$ & $3.36(2-5)$ & 0.419 \\
\hline Cedar pollen (actual value) & $17.2(4.1-39.1)$ & $21.0(1.68-73.2)$ & 0.267 \\
\hline Cypress pollen (class) & $1.19(0-2)$ & $1.36(0-3)$ & 0.283 \\
\hline Cypress pollen (actual value) & $1.00(0-2.98)$ & $1.36(0-4.81)$ & 0.266 \\
\hline HD (class) & $0.625(0-4)$ & $0.182(0-2)$ & 0.1 .36 \\
\hline HD (actual value) & $2.37(0-32.1)$ & $0.12(0-1.41)$ & 0.182 \\
\hline Mite (class) & $0.625(0-4)$ & $0.182(0-2)$ & 0.136 \\
\hline Mite (actual value) & $2.09(0-26.9)$ & $0.145(0-1.59)$ & 0.174 \\
\hline Ragweed (class) & $0.125(0-2)$ & $0.545(0-3)$ & 0.0706 \\
\hline Ragweed (actual value) & $0.0938(0-1.5)$ & $1.25(0-11.7)$ & 0.0985 \\
\hline
\end{tabular}

( $t$-test, *Fisher's exact test)

There were no significant differences between the two groups with regard to age, sex, eosinophil count at diagnosis, total IgE, and the class and actual RAST values for cedar (JC), cypress, house dust (HD), mite, and ragweed. 


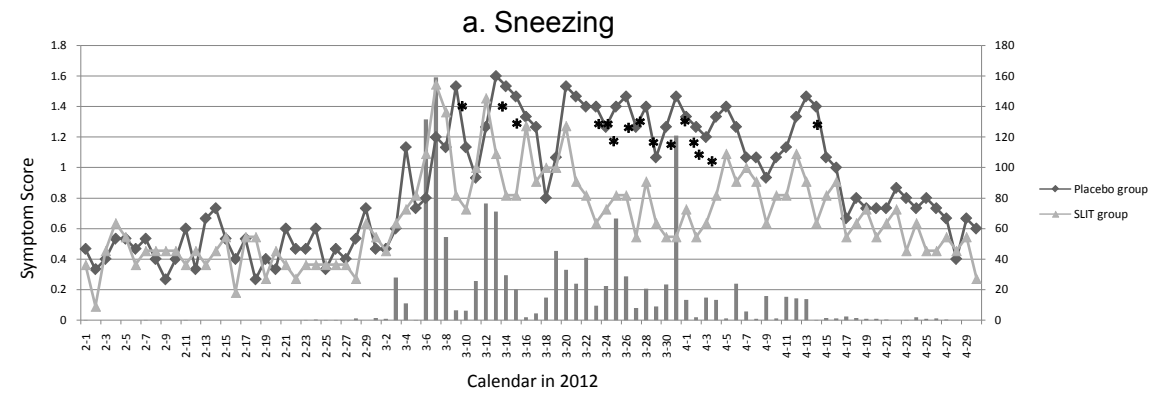

b. Nasal itching
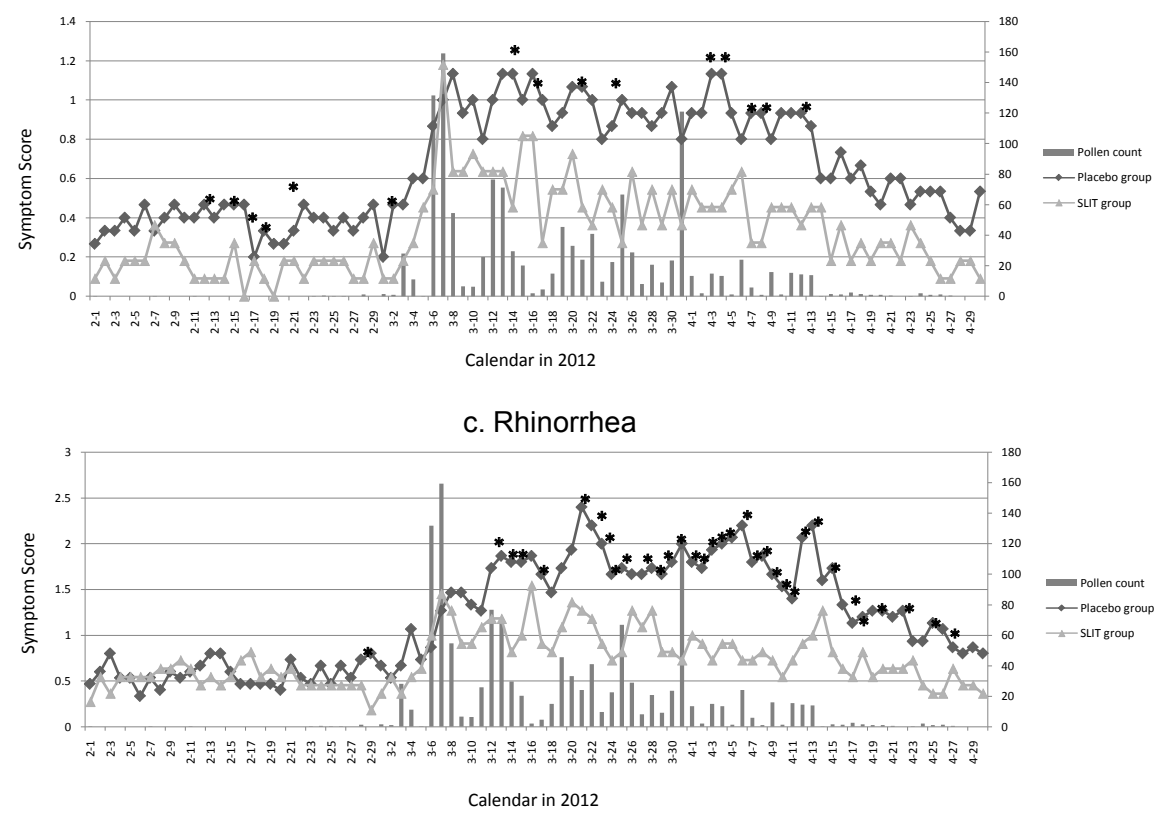

d. Nasal congestion

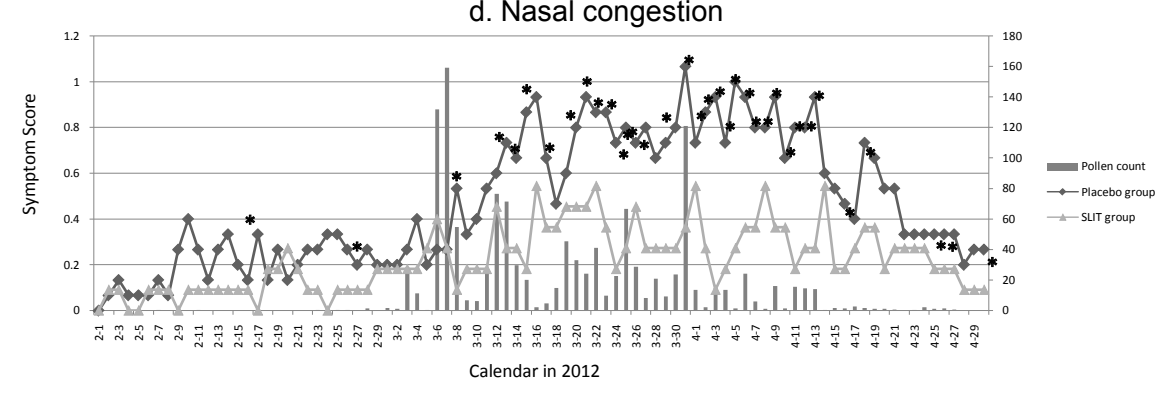

e.TNSS

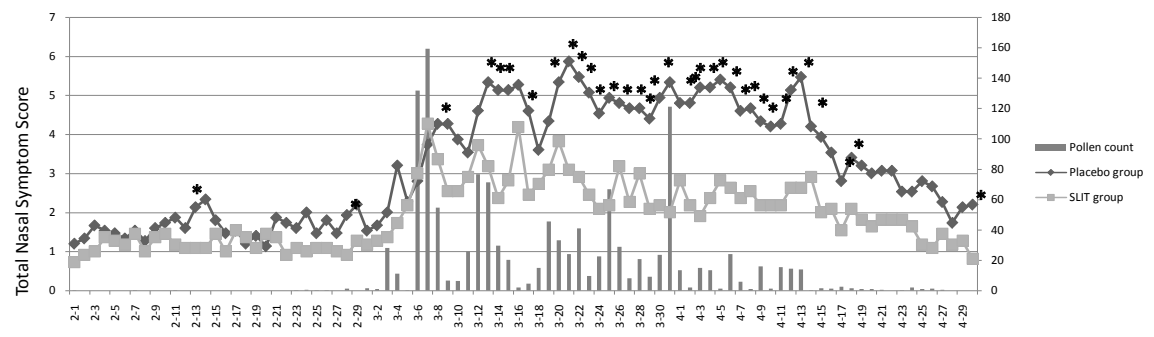

Figure 3. Changes of symptoms

Calendar in 2012

Changes of the mean sneezing score (a), nasal itching score (b), rhinorrhea score (c), nasal congestion score (d), and TNSS (e) in the placebo group and SLIT group. The pollen count is also shown.

*Significant difference. 
the placebo group for a significantly higher percentage of time during the pollen season than during the total observation period.

\section{Adverse events}

Adverse events were observed in 2 subjects from the placebo group $(2 / 16)$ and 5 subjects from the SLIT group (5/12) (Table 3). In the SLIT group, adverse events included oral numbness (2 subjects), oral itching (3 subjects), and cough (in 1 of the 3 subjects with oral itching). All of these events were Grade 1 according to the Common Terminology Criteria for Adverse Events (CTCAE) and resolved spontaneously. In the placebo group, generalized itching and a hot sensation occurred in 1 subject each, but both events resolved before the pollen season. No new adverse events occurred during the pollen season.

\section{Serum levels of cedar allergen-specific IgG, IgG4, and IgE}

Figure 4 shows the serum levels of JC-specific IgG, IgG4, and IgE before treatment and after the pollen season. There was no significant difference of JC-specific IgG between the placebo group and the SLIT group before treatment $(p=0.137)$, but the level was significantly higher in the SLIT group than the placebo group $(p=0.00348)$ after the pollen season. No significant difference of cedar JC-specific IgG4 was observed between the two groups before treatment $(p=0.451)$. Although the JC-specific IgG4 level was higher in the SLIT group than the placebo group after the pollen season, there was still no significant difference $(p=0.0542)$. While there was no significant difference of JC-specific IgE between the two groups before treatment $(p=0.384)$, JC-specific IgE was significantly higher in the SLIT group than the placebo group after the pollen season $(p=0.00624)$. There was no significant difference of the JC-specific IgG4/IgG ratio between the two groups either before treatment ( $p=0.239$ ) or after the pollen season $(p=0.3792)$.

We also examined the changes in the serum levels of JC-specific IgG, IgG4, and IgE from before treatment to after the pollen season (Figure 5). The change of JC-specific IgG was significantly larger in the SLIT group compared with the placebo group $(p=0.00982)$, as was the change of JC-specific IgG4 $(p=0.0122)$. The change of JC-specific IgE was also significantly larger in the SLIT group compared with the placebo group ( $p=0.0214$ ). Furthermore, the change of the IgG4/IgG ratio was significantly larger in the SLIT group than the placebo group $(p=0.00189)$.

\section{Discussion}

Antigen-specific immunotherapy is the only curative treatment for allergic rhinitis. Various methods of antigen administration have been examined, and SCIT has become a popular method that is effective at relatively low allergen doses. In Western countries, SLIT has also come to be widely used instead of SCIT because it is safer, painless, and does not require frequent visits for treatment. The efficacy of SLIT for allergic rhinitis has been demonstrated by a meta-analysis [8], and high dose SLIT is expected to have a similar effect to SCIT while being less invasive.

According to a position paper from the World Health Organization [9], immunotherapy is more effective when started at a younger age. In addition, Des Roches et al. [10] reported that performing SCIT for dust mite allergy in infants with asthma significantly reduced future sensitization to new allergens. Furthermore, Honda et al. [11] reported that giving Japanese infants immunotherapy for house dust allergy inhibited sensitization to cedar pollen.

In Japan, allergic rhinitis (perennial or seasonal) is increasing

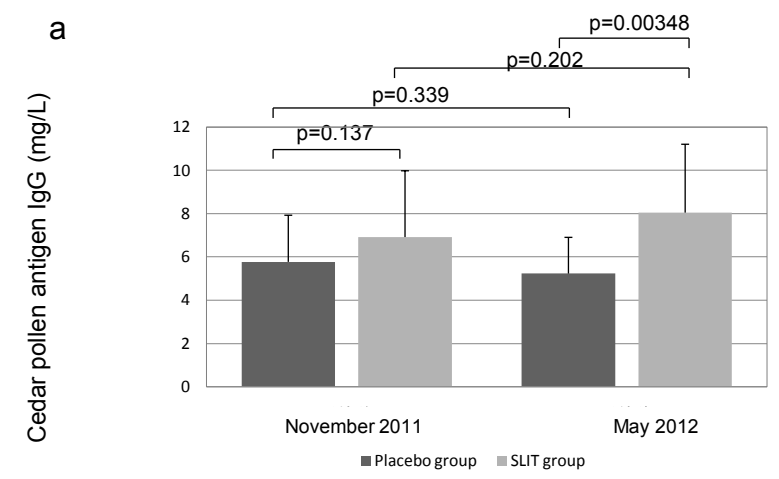

b
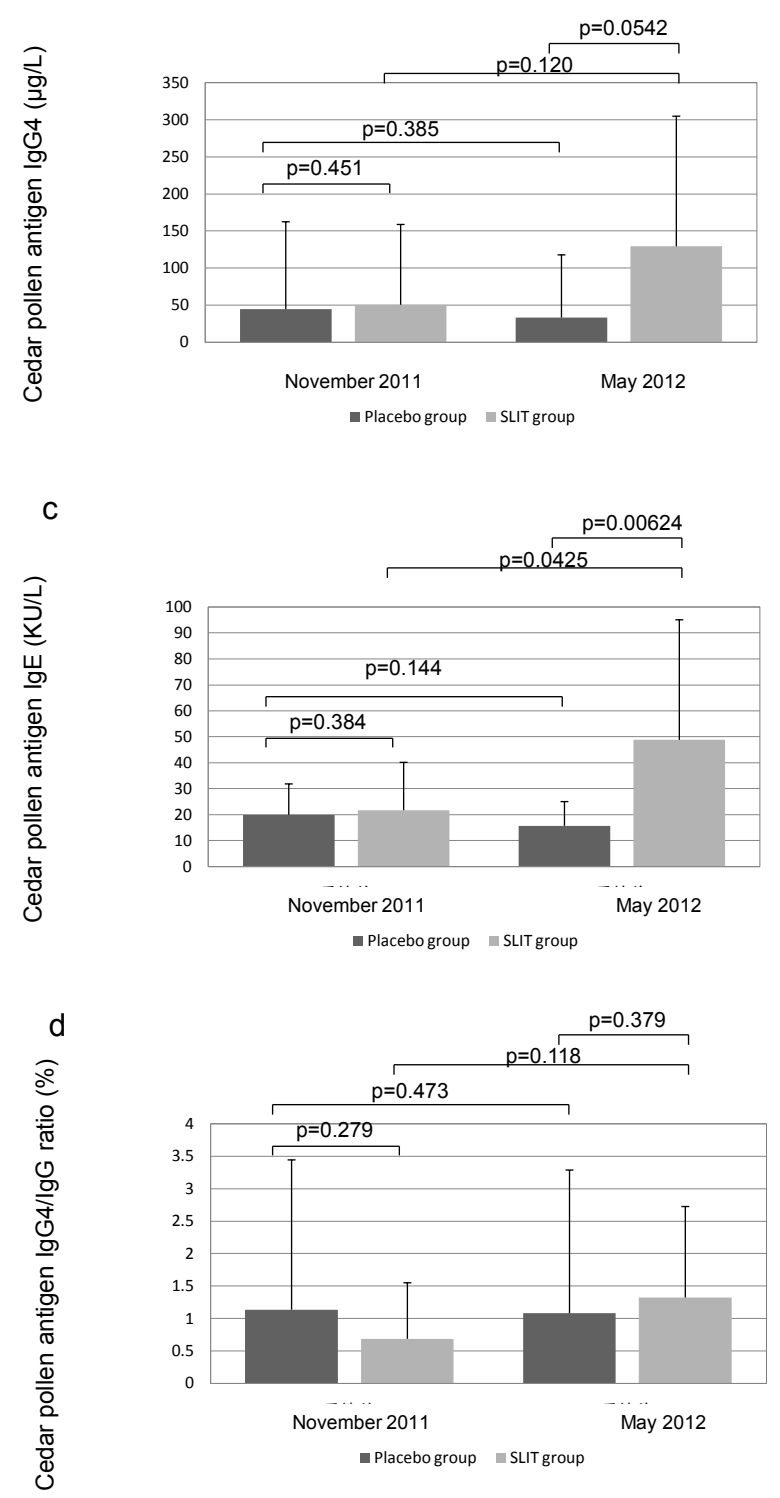

Figure 4. Serum levels of JC-specific $\operatorname{IgG}$ (a), $\operatorname{IgG} 4$ (b), and $\operatorname{IgE}$ (c), as well as the $\operatorname{IgG} 4$ IgG ratio (d), before treatment (November 2011) and after the pollen season (May 2012). IgG and IgE levels were significantly higher in the SLIT group than the placebo group after the pollen season. While JC-specific IgG4 was higher in the SLIT group after the pollen season, the difference was not significant. There was no significant difference of the IgG4/ $\mathrm{IgG}$ ratio between the 2 groups before or after treatment. 
IgG

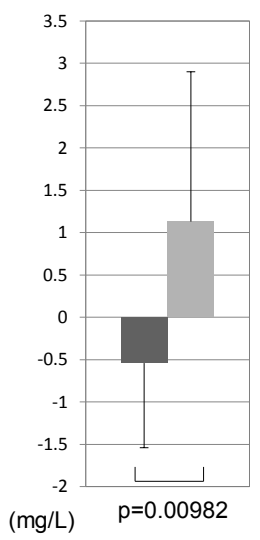

$\operatorname{lgG4}$

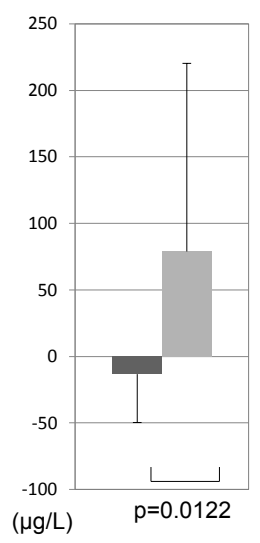

IgE

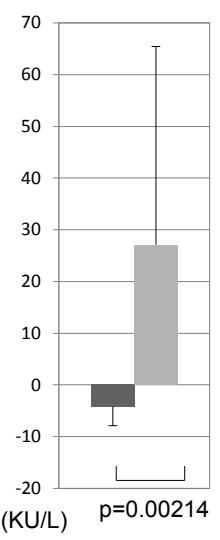

IgG4/IgG

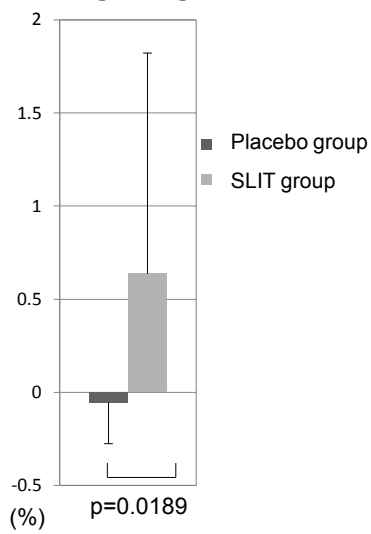

Figure 5. Changes of serum JC-specific IgG, IgG4, IgE, and the IgG4/IgG ratio from before to after treatment in the SLIT group and the placebo group. All variables showed a significant increased after treatment in the SLIT group.

among young people and the causative antigens are becoming more diverse, suggesting that early immunotherapy may be needed for allergic rhinitis. Yuta et al. [12] reported that SLIT was effective from the first year of treatment in infants with JC pollen allergy. Immunotherapy is widely used for JC pollen allergy in Japan, and various parameters have been employed to assess its efficacy. In this study, we employed symptoms and serum levels of JC-specific antibody to assess the therapeutic effect of immunotherapy.

The pollen count was high in 2011, but it was lower than usual in 2012 when this study was performed. In fact, the total pollen count in Chiyoda Ward of Tokyo (the closest location to Nippon Medical School hospital) was approximately $1250 / \mathrm{cm}^{2}$ by the Durham method. In 2005, Okubo et al. [13] investigated SLIT for cedar pollen allergy using a symptom diary to determine scores for symptoms. The pollen count was high that year and the total pollen count in Chiyoda Ward was $10625 / \mathrm{cm}^{2}$. This may have influenced their results, since they reported a higher TNSS in both the SLIT and placebo groups compared with our findings and the score was significantly lower in the SLIT group on only $4 / 43$ days $(9.3 \%)$. In contrast, we found that the TNSS was significantly lower in the SLIT group on $37 / 90$ days (41.1\%). It is possible that this difference in clinical efficacy was related to the lower pollen count in 2012 .

It has been pointed out that clinical evaluation of allergic rhinitis tends to be dependent on subjective information obtained by methods such as symptom scores, quality of life assessment charts, and visual analogue scales. However, this is unavoidable because the main symptoms of allergic rhinitis are sneezing, rhinorrhea, itching, nasal congestion, or ocular symptoms that impair the quality of life, and it is necessary to determine efficacy based on the response of such clinical manifestations. In the present study, we gave the subjects a detailed explanation of how to count the number of sneezing or nose blowing episodes in order to minimize inconsistency.

During the 90-day observation period of the present study, the individual symptom scores and TNSS were significantly lower in the SLIT group than the placebo group for times ranging from 15 to 37 days. During the 42-day JC pollen season (March 3 to April 13), these scores were significantly lower in the SLIT group for 14 to 28 days. Symptoms scores (except for nasal itching) were lower in the SLIT group than the placebo group for a significantly higher percentage of time during the actual pollen season than during the total observation
Table 3. Adverse events

\begin{tabular}{|l|l|l|l|}
\hline & Event & Timing of onset & Duration \\
\hline $\begin{array}{l}\text { Placebo group } \\
(2 / 16)\end{array}$ & Generalized itching & Early up-titration period & Few days \\
\cline { 2 - 4 } & Hot sensation & Early maintenance period & Few days \\
\hline \multirow{4}{*}{$\begin{array}{l}\text { SLIT group } \\
(5 / 12)\end{array}$} & Oral numbness & Early up-titration period & Few days \\
\cline { 2 - 4 } & Oral numbness & Early up-titration period & Few days \\
\cline { 2 - 4 } & Oral itching & $\begin{array}{l}\text { Throughout the up-titration } \\
\text { period }\end{array}$ & $\begin{array}{l}\text { Approximately } 2 \\
\text { weeks }\end{array}$ \\
\cline { 2 - 4 } & Oral itching, cough & Early up-titration period & Few days \\
\hline & Oral itching & Early maintenance period & Few days \\
\hline
\end{tabular}

All adverse events in the SLIT group were Grade 1 according to CTCAE and resolved spontaneously pollen season. Adverse events were also seen in the placebo group and they were resolved before the pollen season.

period. Although this study was conducted in a pollen season with a relatively low total pollen count, the TNSS was significantly decreased for about two thirds of the pollen season (28 days, 66.7\%), suggesting that SLIT was sufficiently effective from the first season of treatment.

It has been reported that the effect of immunotherapy is dose dependent, and that higher doses are required for SLIT to obtain the same therapeutic effect as SCIT [14]. If SCIT was started at the same time with the maintenance dose being 200 JAU/M $(0.1 \mathrm{~mL}$ of $2000 \mathrm{JAU}$ extract) or $600 \mathrm{JAU} / \mathrm{M}(0.3 \mathrm{~mL}$ of $2000 \mathrm{JAU}$ extract $)$, the total allergen dose would be approximately 2000 JAU and 5800 JAU, respectively. Since the dose used for SLIT in our study was 4000 JAU, the total dose administered was approximately $106,400 \mathrm{JAU}$, which is respectively 53.2 and 18.3 times higher than with standard SCIT. SLIT was significantly more effective than placebo in our study, but the relation of therapeutic effect to the dose of cedar allergen needs to be examined in the future.

Adverse effects of SCIT range from injection site symptoms (swelling, redness, and pain) to systemic anaphylaxis. In contrast, the common adverse effects of SLIT are local symptoms such as oral itching and swelling, while anaphylactic symptoms are considered to be extremely rare. In the present study, no adverse events of CTCAE Grade 2 or higher occurred during SLIT, and all events were minor and did not require treatment. Of the five adverse events observed in the SLIT group, 4 occurred in the early up-titration period and 1 occurred in the early maintenance period, but these events only persisted for a few days and resolved spontaneously. This suggests that attention needs to be paid to adverse events during the antigen 
up-titration period, as is the case with SCIT. There was no significant difference in the number of events between the placebo group and the SCIT group ( $p=0.445$, Fisher's exact test). However, the events in the SLIT group were local oral symptoms, whereas patients in the placebo group complained of generalized symptoms. With regard to the safety of cedar allergen extract, Mitobe et al. [15] reported no systemic side effect after sublingual administration to rats at 300 times the dose for humans.

When immunotherapy is performed, a causative antigen is administered at gradually increasing doses in order to reduce the response, with antigen uptake by dendritic cells, induction of regulatory $\mathrm{T}$ cells, and inhibition of specific Th2 cells all being considered important in the mechanism of action. When SLIT is performed, the allergen passes through the oral mucosa to reach dendritic cells in the floor of mouth. Dendritic cells bind the antigen via the high affinity $\mathrm{IgE}$ receptor (FceR I) and induce regulatory $\mathrm{T}$ (Treg) cells through increased production of transforming growth factor (TGF)- $\beta$. Treg cells inhibit activation and proliferation of Th2 cells by producing IL- 10 and TGF- $\beta$, and also induce class switching of B cells with production of blocking antibodies (such as specific IgA and specific IgG4 antibodies), while production of specific IgG antibody is blocked by inhibition of specific Th2 cells and symptoms are suppressed.

Various biomarkers reflecting the therapeutic mechanism of SLIT have been examined. An ideal biomarker is closely associated with clinical manifestations, but the difficulty of specimen collection and measurement also need to be convenient. Samples collected by intranasal irrigation and nasal mucosa brushing cytology vary in volume depending on the sampling time, while biopsy of the nasal mucosa is invasive and difficult to conduct at some centers. On the other hand, serum biomarkers are convenient to measure and obtaining a blood sample is minimally invasive, although such biomarkers may not be completely correlated with manifestations of nasal allergy.

It has been reported that serum IL-10 and TGF- $\beta$ are the biomarkers for allergen-specific SCIT and SLIT for allergic rhinitis $[16,17]$, but these markers are difficult to measure. In contrast, measurement of immunoglobulins is quite feasible. Allergen-specific immunotherapy was reported to increase the blood level of IgG4, and Gleich proposed that allergen-specific IgG4 acts as a blocking antibody [18]. SLIT increases serum levels of antigen-specific IgG4 and also increases the antigen-specific IgG4/IgE ratio $[19,20]$, and it has been suggested that serum IgG acts as blocking antibody and is associated with symptomatic improvement [21]. In Japan, it was reported that allergen-specific SLIT increases serum Cry j 1-specific IgG4 antibody in patients with JC pollinosis [22].

In the present study, we employed an assay kit that allowed measurement of specific IgE with higher precision than the standard method, as well as allowing measurement of serum JC-specific IgG4, which has been previously difficult to measure owing to very low levels. Accordingly, we could evaluate the usefulness of JC-specific IgG4 as a biomarker.

We found that the serum level of JC-specific IgG was significantly higher in the SLIT group than the placebo group after the pollen season, and the change of the specific IgG level from before to after the season was significantly larger in the SLIT group. While JC-specific IgG4 did not show a significant increase in the SLIT group after the pollen season, the change of IgG4 was significantly larger in the SLIT group. Also, the change of the JC-specific IgG4/IgG ratio after treatment was significantly larger in the SLIT group, demonstrating that the proportion of JC-specific IgG4 in IgG was increased by SLIT. This suggested the possible involvement of IgG4, which is considered to be a blocking antibody, in the mechanism of SLIT.

To in investigate the correlation of the clinical effectiveness and the serum levels of immunoglobulins, SLIT group patients were classified in 2 subgroups, including active responders $(n=6)$ and active nonresponders $(n=6)$, but dramatic difference was not detected between active responders and active nonresponders in the changes in the serum levels of JC-specific IgG, IgG4, and IgE and in the IgG4/IgG ratio.(data not shown)

It has been often proposed that reduction of allergen-specific IgE is the mechanism of immunotherapy. It was reported that allergenspecific immunotherapy briefly increases the IgE level and subsequently reduces it. Fujimura et al. reported that SLIT was more effective for JC pollinosis when the serum cedar allergen-specific IgG4/total IgE ratio was low before treatment than when the ratio was high [20]. In the present study, we found a significant increase of allergen-specific IgE after the pollen season in the SLIT group.

\section{Conclusion}

We demonstrated the effectiveness of SLIT for JC pollinosis during a pollen season when the level of pollen dispersion was suitable for determining the clinical efficacy. By measuring serum levels of JCspecific IgG and IgG4, we also demonstrated an increase of the JCspecific IgG4/IgG ratio in the SLIT group after the pollen season, suggesting that IgG4 may be involved in the therapeutic mechanism of SLIT. If SLIT becomes more popular for treating cedar pollen allergy in Japan, biomarkers that can predict the response will be needed. Serum levels of JC-specific IgG and IgG4 can be measured with a specific IgE assay kit at most hospitals, suggesting that these antibodies can be used as biomarkers.

\section{References}

1. Blackley CH (1873) Hay-fever: its causes, treatment, and effective prevention. Bailliere.

2. Horiguchi S, Saito Y (1964) [Discovery of Japanese Cedar Pollinosis In Nikko, Ibaraki Prefecture]. Arerugi 13: 16-18. [Crossref]

3. Baba K, Nakae K (2008) National epidemiological survey of flower allergy 2008 (comparison with 1998 results) involving otolaryngologists and their family members. Prog Med 28: 2001-2012.

4. Noon L (1911) Prophylactic inoculation against hayfever. Lancet 1:1572.

5. Klimek L, Dormann D, Jarman ER, Cromwell O, Riechelmann H, Reske-Kunz AB (1999) Short-term preseasonal birch pollen allergoid immunotherapy influences symptoms, specific nasal provocation and cytokine levels in nasal secretions, but not peripheral T-cell responses, in patients with allergic rhinitis. Clin Exp Allergy 29: 13261335 [Crossref]

6. Pastorello EA, Ortolani C, Incorvaia C, Farioli L, Italia M, et al. (1990) A double-blind study of hyposensitization with an alginate-conjugated extract of Dermatophagoides pteronyssinus (Conjuvac) in patients with perennial rhinitis. II. Immunological aspects. Allergy 45: 505-514. [Crossref]

7. Okubo K, Gotoh M (2009) Sublingual immunotherapy for Japanese cedar pollinosis Allergol Int 58: 149-154. [Crossref]

8. Wilson DR, Lima MT, Durham SR (2005) Sublingual immunotherapy for allergic rhinitis: systematic review and meta-analysis. Allergy 60: 4-12. [Crossref]

9. Bousquet J, Lockey R, Malling HJ (1998) Allergen immunotherapy: therapeutic vaccines for allergic diseases. A WHO position paper. J Allergy Clin Immunol 102 558-562. [Crossref]

10. Des Roches A, Paradis L, Menardo JL, Bouges S, Daurés JP, Bousquet J (1997) Immunotherapy with a standardized Dermatophagoides pteronyssinus extract. VI. Specific immunotherapy prevents the onset of new sensitizations in children. $J$ Allergy Clin Immunol 99: 450-453. [Crossref] 
11. Honda K, Asaka C, Fukui N, et al. (2010) Long-term allergen-specific immunotherapy results in allergic rhinitis. Practica Otologica 129: 64-67.

12. Yuta A, Miyamoto Y, Ogihara H, Hattori R, Okubo K (2009) [Antigen specific sublingual immunotherapy for pediatric Japanese cedar pollinosis]. Arerugi 58: 124132. [Crossref]

13. Okubo K, Gotoh M, Fujieda S, Okano M, Yoshida H, et al. (2008) A randomized double-blind comparative study of sublingual immunotherapy for cedar pollinosis. Allergol Int 57: 265-275. [crossref]

14. Di Bona D, Plaia A, Leto-Barone MS, La Piana S, Di Lorenzo G (2012) Efficacy of subcutaneous and sublingual immunotherapy with grass allergens for seasonal allergic rhinitis: a meta-analysis-based comparison. J Allergy Clin Immunol 135: 1097-1105. [Crossref]

15. Mitobe Y, Yokomoto Y, Ohashi-Doi K (2015) Safety evalution of standardized allergy extract of Japanese cedar pollen dor sublingual immunotherapy. Regul Toxicol Pharmacol 71: 529-540. [Crossref]

16. Piconi S, Trabattoni D, Rainone V, Borgonovo L, Passerini S, et al. (2010) Immunological effects of sublingual immunotherapy: clinical efficacy is associated with modulation of programmed cell death ligand 1, IL-10, and IgG4. J Immunol 185: 7723-7730. [Crossref]

17. Ciprandi G, De Amici M, Tosca MA, Pistorio A, Marseglia GL (2009) Sublingual immunotherapy affects specific antibody and TGF-beta serum levels in patients with allergic rhinitis. Int J Immunopathol Pharmacol 22: 1089-1096. [Crossref]
18. Gleich GJ, Zimmermann EM, Henderson LL, Yunginger JW (1982) Effect of immunotherapy on immunoglobulin $\mathrm{E}$ and immunoglobulin $\mathrm{G}$ antibodies to ragweed antigens: a six-year prospective study. J Allergy Clin Immunol 70: 261-271. [Crossref]

19. La Rosa M, Ranno C, André C, Carat F, Tosca MA, Canonica GW (1999) Doubleblind placebo-controlled evaluation of sublingual-swallow immunotherapy with standardized parietaria judaica extract in children with allergic rhinoconjunctivitis. $J$ Allergy Clin Immunol 104: 425-432. [Crossref]

20. Pajno GB, Morabito L, Barberio G, Parmiani S (2000) Clinical and immunologic effects of long-term sublingual immunotherapy in asthmatic children sensitized to mites: a double-blind, placebo-controlled study. Allergy 55: 842-849. [Crossref]

21. Lima MT, Wilson D, Pitkin L, Roberts A, Nouri-Aria K, et al. (2002) Grass pollen sublingual immunotherapy for seasonal rhinoconjunctivitis: a randomized controlled trial. Clin Exp Allergy 32: 507-514. [Crossref]

22. Horiguchi S, Okamoto Y, Yonekura S, Okawa T, Yamamoto H, et al. (2008) A randomized controlled trial of sublingual immunotherapy for Japanese cedar pollinosis. Int Arch Allergy Immunol 146: 76-84. [Crossref]

23. Fujimura T, Yonekura S, Horiguchi S, Taniguchi Y, Saito A, et al. (2011) Increase of regulatory $\mathrm{T}$ cells and the ratio of specific $\operatorname{IgE}$ to total $\operatorname{IgE}$ are candidates for response monitoring or prognostic biomarkers in 2-year sublingual immunotherapy (SLIT) for Japanese cedar pollinosis. Clin Immunol 139: 65-74. [Crossref]

Copyright: $(02016$ Masuno S. This is an open-access article distributed under the terms of the Creative Commons Attribution License, which permits unrestricted use, distribution, and reproduction in any medium, provided the original author and source are credited. 